\title{
Análises estatísticas, visuais e não paramétricas para a otimização do ajuste de curvas IDF e construção de ábacos de projeto de obras hidráulicas: estudo de caso em São Carlos - SP
}

\section{Statistical, visual and nonparametric analysis for optimization of IDF curves and design aid charts for hydraulic works: study case in Sao Carlos - SP}

\author{
Data de entrada: \\ 07/11/2019 \\ Data de aprovação: \\ 04/12/2019
}

Marcus Nóbrega Gomes Júnior ${ }^{1 *}$ | Pedro Henrique Alves Braga ${ }^{1}$ | Eduardo Mario Mendiondo ${ }^{1}$ | Luisa Fernanda Ribeiro Reis ${ }^{1}$

DOI: https://doi.org/10.36659/dae.2021.013

ORCID ID

Gomes Júnior MN https://orcid.org/0000-0002-8250-8195

Braga PHA (iD https://orcid.org/0000-0003-4333-488X
Mendiondo EM (D) htps://orcid.org/0000-0003-2319-2773

Reis LFR Dttps://orcid.org/0000-0003-4937-5404

\section{Resumo}

Um dos elementos mais importantes no projeto de obras de drenagem urbana para o controle de cheias é a intensidade máxima média de precipitação para dada duração e probabilidade anual. Tipicamente, essa informação é apresentada no formato de Curvas de Intensidade - Duração e Frequência (IDF). Porém a frequente ausência de séries extensas de precipitação leva à necessidade de extrapolações estatísticas via distribuições teóricas de probabilidade. Decidir qual é a melhor distribuição de probabilidade que explique as observações e permita a extrapolação para maiores tempos de retorno depende das observações de precipitação e de sua qualidade. Desse modo, o objetivo desse artigo é avaliar via análises estatísticas (Coeficiente de Determinação) não paramétricas (teste de Kolmogorov-Smirnov e $P$-Valor) e visuais as melhores combinações entre os métodos de avaliação de frequência empíricos (Weibull, Mediana, Hosking, Blow, Cunnane, Gringorten, Hazen, Tukey's e Chegodayev's) e teóricos (Gumbel, Log-Normal e Normal) para aos dados históricos de São Carlos - SP (1961-2018). Com a IDF obtida pelo ajuste otimizado, ábacos foram construídos utilizando o método racional modificado como uma forma de elaborar cálculos rápidos de vazões de pico para pequenas bacias.

Palavras-chave: Distribuição de Probabilidade de Gumbel. Weibull. Kolmogorov-Smirnov. IDF.

\section{Abstract}

One of the most essential design parameters for the designing of flood control hydraulic drainage structures is the average maximum precipitation intensity for a specific rainfall duration. Typically, this information is provided by Intensity-Duration-Frequency curves. However, the usual absence of long series of precipitation requires the use of statistical extrapolations by theoretical probability distributions. Deciding the optimal probability distribution

\footnotetext{
${ }^{1}$ Universidade de São Paulo - Escola de Engenharia de São Carlos - São Paulo - Brasil.

*Autor correspondente: marcusnobrega.engcivilagmail.com.
} 
that explains observations and efficiently enables extrapolations to relatively larger return periods depends on the available precipitation data and its data quality. Thus, the objective of this paper is to (a) assess by statistical analyses (Determination Coefficient), nonparametric analyses (Kolgomov-Smirnov test and P-Value) and visual analyses the optimized combinations among some empirical frequency distribution formulae (Weibull, Mediana, Hosking, Blow, Cunnane, Gringorten, Hazen, Tukey's e Chegodayev's) and Theoretical (Gumbel, Log-Normal e Normal). This methodology is applied for the available historical precipitation data at São Carlos - SP (1961-2018). With the optimized adjustment, an IDF curve was designed and (b) maximum peak flow charts were plotted to guide the pre-design of hydraulic drainage structures in small watersheds using the modified rational method.

Keywords: Gumbel probability distribution. Weibull. Kolmogorov-Smirnov test. IDF.

\section{INTRODUÇÃO}

A alteração da cobertura vegetal promovida pela urbanização impacta a infiltração, alterando o processo de transformação de chuva em escoamento superficial em bacias urbanizadas. $\mathrm{O}$ aumento dos escoamentos superficiais em função da diminuição das resistências hidráulicas pela organização dos escoamentos, gerando canais preferenciais, ocasiona o acúmulo rápido dos escoamentos em pontos críticos na bacia hidrográfica. Com menores tempos de percurso da onda de cheia, aumentam as chances de inundações ou alagamentos. Assim, os projetos de drenagem urbana de canais, calhas, condutos e/ou outras medidas mitigadoras de drenagem urbana devem considerar eventos críticos, especialmente em bacias urbanizadas.

Portanto, é prática corrente na elaboração de projetos de drenagem urbana a utilização de curvas Intensidade-Duração-Frequência (IDF). A construção dessas curvas, a partir de dados de intensidades de curtos intervalos de tempo, no Brasil, é a forma que introduz menos erros pela maior quantidade de dados disponíveis. Entretanto, a dificuldade de obtenção desses dados, que usualmente são aferidos por pluviógrafos, envolve a sua rara disponibilização em plataformas públicas. Por outro lado, dados de precipitações diárias, isto é, aferidos por pluviômetros, possuem ampla divulgação. Em projetos de drenagem urbana, es- pecialmente em cidades com altas taxas de impermeabilização, as chuvas de menor duração e maior intensidade são mais importantes do que chuvas diárias no que tange ao dimensionamento de medidas mitigadoras de drenagem urbana (MIGUEZ; REZENDE; VERÓL, 2016).

Logo, a utilização de dados diários de precipitação para construir curvas IDF, fazendo o uso de coeficientes de desagregação, tornou-se uma prática corrente (CETESB, 1979; TORRICO, 1974). Batista, Alves e Tavares (2018) desenvolveram equações IDF para 19 municípios da região metropolitana de Fortaleza - CE, utilizando os coeficientes propostos por Torrico (1974). Já Cavalcanti, Silva e Reis (2015) utilizaram coeficientes propostos pela Cetesb (1979) para atualizar a Equação IDF de São Carlos - SP. Outros exemplos de coeficientes de desagregação são os de Silveira (2000) e Back, Oliveira e Henn (2012), que ajustaram coeficientes para dados regionais do Rio Grande do Sul e para o Estado de Santa Catarina, respectivamente.

De acordo com Sane et al., (2018), a incerteza relacionada à estimativa de máximos anuais para períodos de retorno superiores aos dados observados é de 3 a 4 vezes maior que a incerteza relacionada aos coeficientes de desagregação. Portanto, a construção de equações IDF, utilizando 
esses coeficientes, é razoável e aplicável onde não são disponíveis dados subdiários de precipitação.

Em relação às distribuições de probabilidade, diversos estudos foram elaborados nos últimos anos. Gandini e Queiroz (2016) avaliaram o ajuste de 6 métodos de distribuições de probabilidade (Normal, Log-Normal, Pearson tipo III, Log-Pearson tipo III, Gumbel Teórica e Gumbel Finita) aplicados a 21 estações pluviométricas na cidade de Cunha-SP e região. Os autores verificaram o ajuste das distribuições aos dados de precipitação, isto é, baseando-se nos indicadores de ajuste de Kolmogorov-Smirnov e no P-Valor. Foi possível concluir que, para os dados observados, a distribuição Gumbel Finita foi a que obteve melhor aderência.

Uma das hipóteses que norteiam a construção de curvas IDFs é a consideração da estacionaridade da variável aleatória contínua de análise, isto é, a precipitação. Porém alguns modelos de mudanças climáticas como o HadGEM2-ES (JONES et al., 2011) e MIROC5 (WATANABE et al., 2010) indicam que isso pode não ocorrer, o que pode ser explicado pelo deslocamento e escala da curva de distribuição de probabilidade ao longo da faixa de dados observados. É possível obter dados de projeção climática pelo Representative Concentration Pathway (RCP) para diversos cenários. Destacam-se, de acordo com Lago (2018), os cenários RCP 4.5, mais otimista e o cenário RCP 8.5, mais pessimista.

Adib e Ghafari Rad (2017) desenvolveram um método integrado de geração de curvas IDF para cenários de mudanças climáticas. Os autores aplicaram o método em Baghmalek há 40 anos de dados observados no sudeste do Irã (1974-2013); 9 anos de dados extrapolados a partir dos dados medidos para os anos de 2013-2021, via ajustes de probabilidade e 30 anos de dados (2021-2050) modelados utilizando o modelo de projeção climática HadCM3. Foram avaliados 3 cenários distintos de projeção climática. Os autores construíram curvas
IDF com os máximos anuais distribuídos via Gumbel e Log-Pearson tipo III. Foi possível concluir que haverá um aumento de intensidades para períodos de retorno inferiores a 2,33 anos e diminuição para períodos superiores.

No entanto, os recentes estudos avaliaram apenas a distribuição de probabilidade teórica mais adequada para suas amostras. A análise de frequência, utilizando métodos empíricos, ou seja, aquela que se aplicada na amostra determina o tempo de retorno empírico, nem sempre é avaliada. Por exemplo, comparando-se os métodos de frequência empíricos de Weibull, Mediana, Hosking, Blom, Cunnane, Gringorten, Hazen, Tukey's e Chegodayev's, em relação às distribuições teóricas de probabilidade Normal, Log-Normal, e Gumbel, combinações diferentes podem produzir melhores ajustes (Collischonn e Dornelles, 2013). Guo (1990) avaliou o ajuste de diferentes métodos de frequência empíricos para amostras geradas aleatoriamente via criação de séries por Monte Carlo e chegou a bons resultados para as fórmulas de Cunnane e Gringortten.

\section{OBJETIVO}

O objetivo deste trabalho é avaliar estatisticamente as melhores combinações de probabilidades observadas $x$ teóricas que produzam os melhores valores de $\mathrm{P}$-Valor, aceitando o teste de Kolmogorov-Smirnov e de coeficiente de determinação $\left(R^{2}\right)$. Com a melhor combinação obtida, será atualizada a curva IDF de São Carlos e sua utilização servirá para a construção de ábacos de determinação de vazões de projeto para obras hidráulicas em pequenas bacias.

\section{MATERIAL E MÉTODOS}

Na Fig. 1 é possível observar a estrutura de organização do artigo, que é pautado em (1) avaliar a melhor combinação de distribuições de frequên- 
cia empíricas com teóricas; (2) construir a curva IDF de São Carlos com dados atualizados; e (3), em função da nova curva IDF, construir ábacos de vazões máximas de projeto para pequenas bacias.

\subsection{Estudo de Caso}

O município de São Carlos está localizado na região central do estado de São Paulo, possuindo uma área de aproximadamente $1.132 \mathrm{~km}^{2}$, sendo $6 \%$ de área urbana (249.000 hab.). Possui altitudes que variam entre 520 e 1.000 metros, com média de 832 metros. A cidade possui um clima temperado de altitude, com verão chuvoso e inverno seco (IBGE, 2019). A precipitação média anual da ordem de $1560 \pm 260 \mathrm{~mm}$, a temperatura máxima observada ocorreu no dia 19/10/2014, chegando a $37.9^{\circ}$ (dados disponíveis desde 1961; INMET, 2019), a pressão atmosférica média é da ordem de $0.908 \pm 0.00024$ ATM e a pressão atmosférica máxima é da ordem de $0.919 \pm 0.00024$ ATM (INMET, 2019).

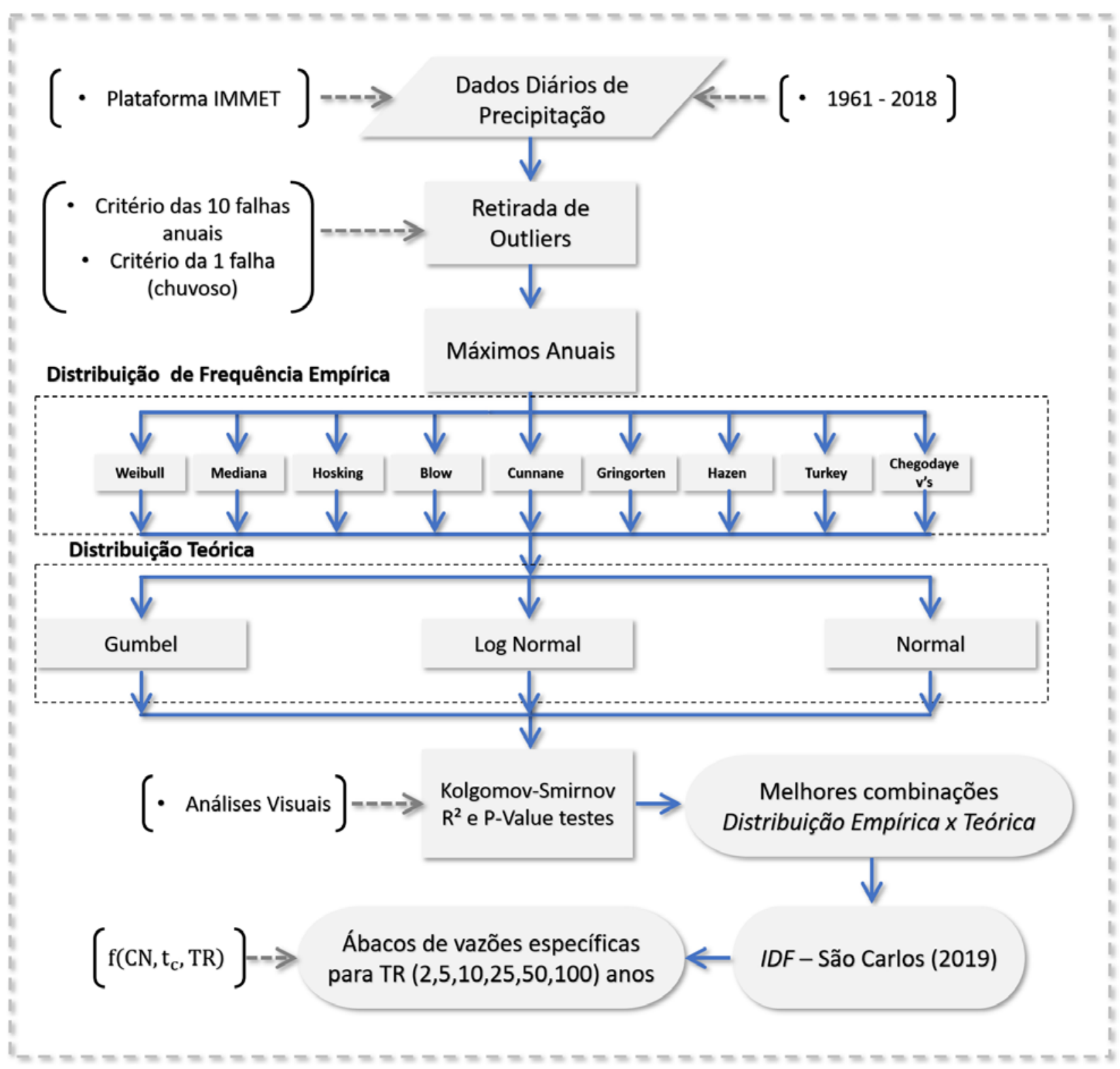

Figura 1 - Estruturação do artigo incluindo métodos utilizados, intervalo da série de dados e tipos de análise elaboradas 
Os dados diários de precipitação para a cidade de São Carlos - SP foram obtidos na plataforma do IMMET (2019), estação No 83.726. Desse modo, para valores de chuvas intensas, foram utilizados coeficientes de desagregação para estimar os valores subdiários (CETESB, 1979). A Fig. 2 possibilita uma análise visual rápida do regime de precipitações local.

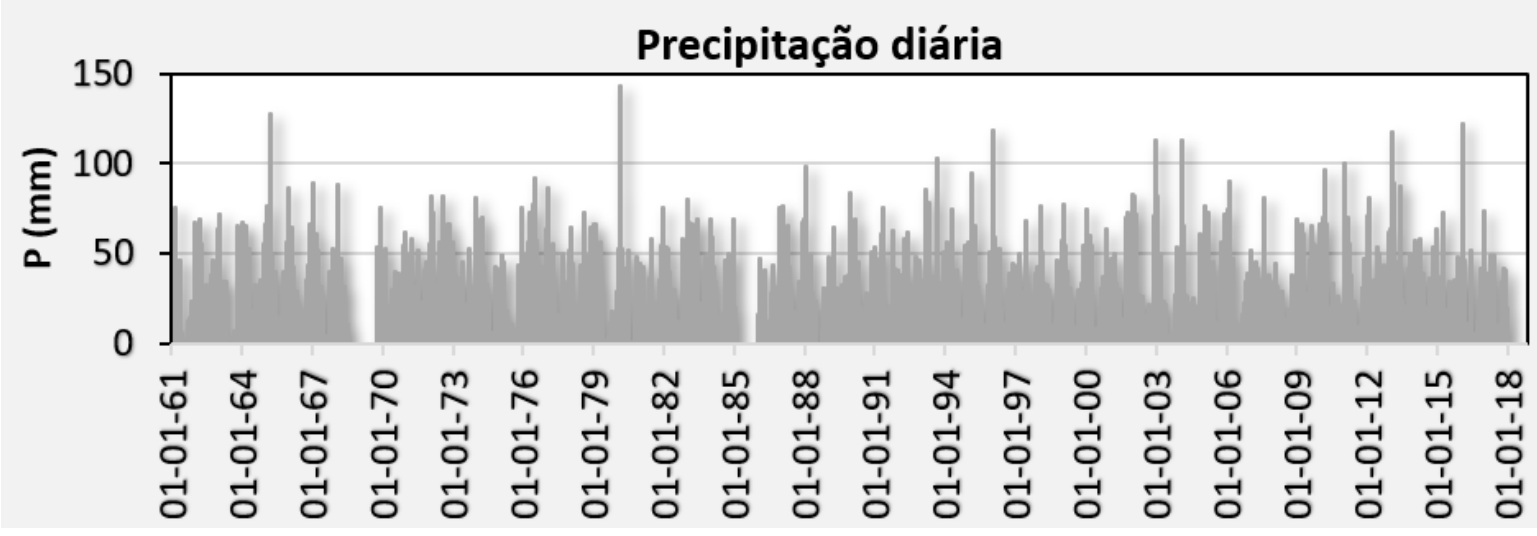

Figura 2 - Dados Históricos de precipitação da cidade de São Carlos - SP.

Fonte: INMET (2019).

\subsection{Remoção de Outliers}

Foi utilizada a metodologia proposta por Britto e Kellner (2016) para a remoção de outliers. Foram removidos anos com mais de 10 falhas ao longo de todo o ano e/ou anos com pelo menos uma faIha no período chuvoso da região, que se inicia em outubro e termina em março. Com os dados tratados, foram selecionadas as precipitações máximas anuais para verificação de outliers pelo Inter Quartil Range (IQR). O IQQR é calculado pela Eq. 1:

$\mathrm{IQR}=\left(\mathrm{Q}_{3}-\mathrm{Q}_{1}\right)$

Em que: $\mathrm{Q}_{1}$ é a precipitação anual correspondente ao percentil $25 \%, Q_{3}$ é a precipitação anual correspondente ao percentil $75 \%$, ambos quando os dados são ordenados de forma crescente.

Os limites superiores $\left(\mathrm{L}_{\mathrm{s}}\right)$ e inferiores $\left(\mathrm{L}_{\mathrm{i}}\right)$ de outliers são dados pelas Eq. 2 e 3, respectivamente:

$\mathrm{L}_{\mathrm{s}}=1,5 \times \mathrm{IQR}+\mathrm{Q}_{3}$
$\mathrm{L}_{\mathrm{i}}=1,5 \times \mathrm{IQR}-\mathrm{Q}_{1}$

Apenas os dados de precipitação máxima anual que estão entre os limites máximo e mínimo são utilizados para construção da IDF.

\subsection{Distribuiç̧ões de frequência empírica}

Segundo Chow (1988), a frequência relativa empírica, ou plotting position, refere-se ao valor atribuído à frequência de probabilidade de cada dado, muito embora às vezes não haja uma quantidade razoável de dados disponíveis. Diversos métodos de frequência empíricas foram propostos, dentre eles Weibull, Mediana, Hosking, Blom, Cunnane, Gringorten, Hazen, Tukey's e Chegodayev's. Se $n$ é o número total de dados e $m$ é a posição desse dado, quando todos os dados são classificados do maior para o menor, a frequência de probabilidade de $m$-ésimo valor de uma amostra grande (n) é: 
$\mathrm{P}\left(\mathrm{X} \geq \mathrm{x}_{\mathrm{m}}\right)=\frac{\mathrm{m}}{\mathrm{n}}$

A Eq. 4 é conhecida como Equação Califórnia. Uma limitação é apresentar uma probabilidade de $100 \%$ quando $m=n$, o que pode ser grosseiro para pequenas amostras e dificulta a plotagem em papéis de probabilidade. Por isso, diversos autores adotaram uma correção na Eq. 4, de modo a torná-la possível de plotar em papéis de probabilidade e evitar limites superiores de pro- babilidade. A maioria das equações empíricas de probabilidade pode ser expressa pela Eq. 5 (CHOW, 1988):

$P\left(X \geq x_{m}\right)=\frac{m-b}{n+1-2 b}$

Na Eq. 5, b é um parâmetro de ajuste. A Tabela 1 mostra diversos valores de $b$ adotados pelos autores e sintetiza a sua fundamentação teórica.

Tabela 1 - Distribuições de frequência empíricas, referencial teórico e contextualização

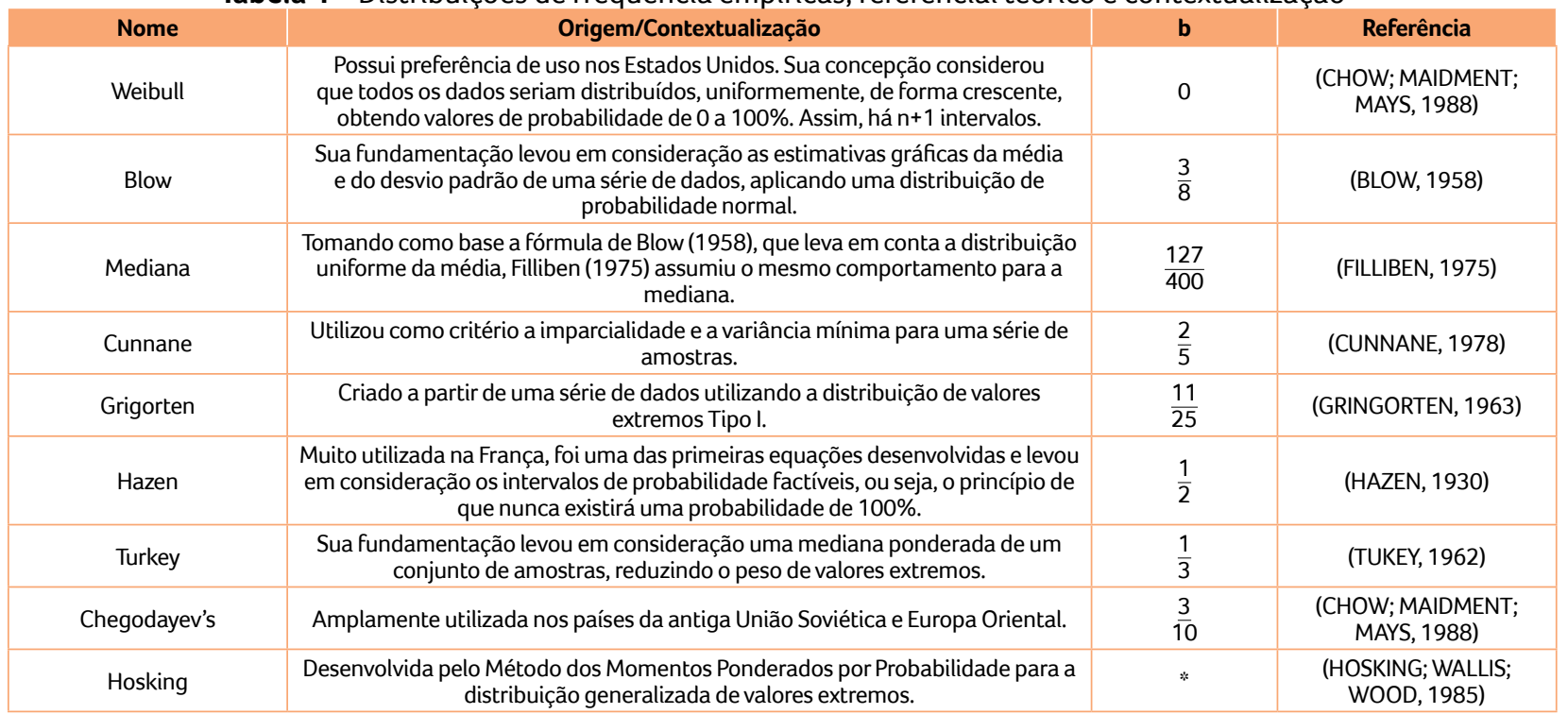

Obs.: A Equação de Hosking (Eq. 6) é a única que não toma por base a Eq. 5, sendo:

$\mathrm{P}\left(\mathrm{X} \geq \mathrm{x}_{\mathrm{m}}\right)=\frac{\mathrm{m}-0.35}{\mathrm{n}}$

\subsection{Distribuições de Probabilidades Teóricas}

\subsubsection{Distribuição Normal}

A distribuição normal considerada tem por base uma variável padronizada $z$. Ela pode ser cumulativa ou em massa. A função de densidade de probabilidade normal $F(x)$ é calculada em função da média e o desvio padrão (Eq. 7). Para determinar a frequência de observações de determinada faixa, multiplica-se a probabilidade de ocorrência pelo número de eventos (Eq. 8) (COLLISCHONN; DORNELLES, 2013).

$$
F(x)=\int_{-\infty}^{x_{i}} \frac{1}{\sqrt{2 \pi} \cdot \sigma_{x}} \cdot e^{-\frac{1}{2}\left(\frac{x_{i}-\bar{x}}{\sigma_{x}}\right)^{2}} d x
$$

Em que: $F(x)$ é a função de densidade de probabilidade acumulada; $x_{i}$ é um dado da amostra; $\sigma_{x}$ é o desvio padrão da amostra; $\bar{x}$ é a média da amostra. 


$$
\mathrm{x}=\overline{\mathrm{x}}+\sigma_{\mathrm{x}} \times \mathrm{K}
$$

Em que: $x$ é a precipitação máxima para uma dada probabilidade; $\bar{x}$ é a média das precipitações máximas anuais; $\sigma_{x}$ é o desvio padrão das precipitações máximas anuais e $\mathrm{K}$ é um fator de frequência da distribuição normal.

\subsubsection{Distribuição Log-Normal}

A distribuição Log Normal (Eq. 9) considera que os logaritmos das precipitações podem ser distribuídos normalmente. Desse modo, ela é bem semelhante à distribuição normal. (COLLISCHONN; DORNELLES, 2013).

$$
\log (\mathrm{x})=\overline{\log (\mathrm{x})}+K \sigma_{\log (\mathrm{x})}
$$

Em que: $\log (\mathrm{x})$ é o logaritmo da precipitação máxima; $\overline{\log (\mathrm{x})}$ é a média dos logaritmos das precipitações máximas anuais observadas; $\sigma_{\log (x)}$ é o desvio padrão dos logaritmos das precipitações máximas anuais observadas e $\mathrm{K}$ é um fator de frequência da distribuição log-normal.

\subsubsection{Distribuição de Gumbel}

A distribuição de Gumbel é descrita pelo desvio padrão e pela média da amostra. Trabalhando as equações, é possível obter, em função do risco ou do tempo de retorno adotado, os valores estimados pela distribuição (Eq. 10) (COLLISCHONN; DORNELLES, 2013).

$$
P=1-e^{-e^{-b}}
$$

Em que: $\mathrm{P}$ é a probabilidade de excedência; $\mathrm{b}$ é dado por:

$$
b=\frac{1}{0,7797 \times s}\left(x-\bar{x}+0,45 \times \sigma_{x}\right)
$$

Em que: x é a precipitação máxima anual; $\sigma_{x}$ é o desvio padrão das precipitações máximas anuais; $\bar{x}$ é a média das precipitações máximas anuais.

Combinando as equações 10 e 11, obtém-se a Eq. 12.

$$
\mathrm{x}=\overline{\mathrm{x}}-\sigma_{\mathrm{x}}\left\{0,45-0,7797 \ln \left[\ln \left(\frac{\mathrm{TR}}{\mathrm{TR}-1}\right)\right]\right\}
$$

Em que: x é a precipitação máxima anual; $\sigma_{x}$ é o desvio padrão das precipitações máximas anuais; $\bar{x}$ é a média das precipitações máximas anuais; TR é o tempo de retorno em anos, dado pelo inverso da probabilidade de excedência.

\subsection{Testes de aderência}

Foram utilizados três testes de aderência (Kolmogorov-Smirnov, P-Valor e Coeficiente de determinação) para verificar as melhores combinações de distribuições de frequência empíricas e teóricas.

\subsubsection{Kolmogorov-Smirnov}

$$
\begin{aligned}
& \mathrm{D}=\operatorname{máx}\left[\left(\operatorname{máx}_{-\infty<\mathrm{x}<\infty}\left|\mathrm{S}_{\mathrm{N}}(\mathrm{x})-\mathrm{P}(\mathrm{x})\right|\right) ;\right. \\
& \left.\left(\operatorname{máx}_{-\infty<\mathrm{x}<\infty}\left|\mathrm{S}_{\mathrm{N}_{1}}(\mathrm{x})-\mathrm{S}_{\mathrm{N}_{2}}(\mathrm{x})\right|\right)\right]
\end{aligned}
$$

Em que: $S_{N}(x)$ é a função de distribuição de frequência empírica acumulada; $P(x)$ é a função cumulativa de distribuição de probabilidade teórica (CDF) e $\mathrm{S}_{\mathrm{N} 1}$ e $\mathrm{S}_{\mathrm{N} 2}$ são dois pontos adjacentes da curva $C D F$. 
A função de densidade de probabilidade acumulada CDF da distribuição K-S é dada de acordo com Press et al. (2007) pela Eq. 14.

$$
P_{K S}=\frac{\sqrt{2 \pi}}{z} \sum_{j=1}^{\infty} \exp \left(-\frac{(2 j-1)^{2} \pi^{2}}{8 z^{2}}\right)
$$

Onde z (Eq. 15) é a variável que descreve a distribuição e $\mathrm{P}_{\mathrm{KS}}$ é a probabilidade negar a hipótese nula, que assume que as distribuições empíricas e teóricas são iguais estatisticamente.

$$
\mathrm{z}=\left(\sqrt{\mathrm{N}_{\mathrm{e}}}+0,12+\frac{0,11}{\sqrt{\mathrm{N}_{\mathrm{e}}}}\right) \mathrm{D}
$$

Em que: $\mathrm{N}_{\mathrm{e}}$ é o tamanho efetivo da amostra que assintoticamente tende ao número total das amostras para valores maiores ou iguais a 4 (PRESS et al., 2007); e D é a estatística de Kolmogorov-Smirnov.

A Fig. 3 representa o gráfico de P-Valor em função da variável descritiva $z$ e da estatística de Kolmogorov-Smirnov para distintos tamanhos de amostra e para 39 elementos.

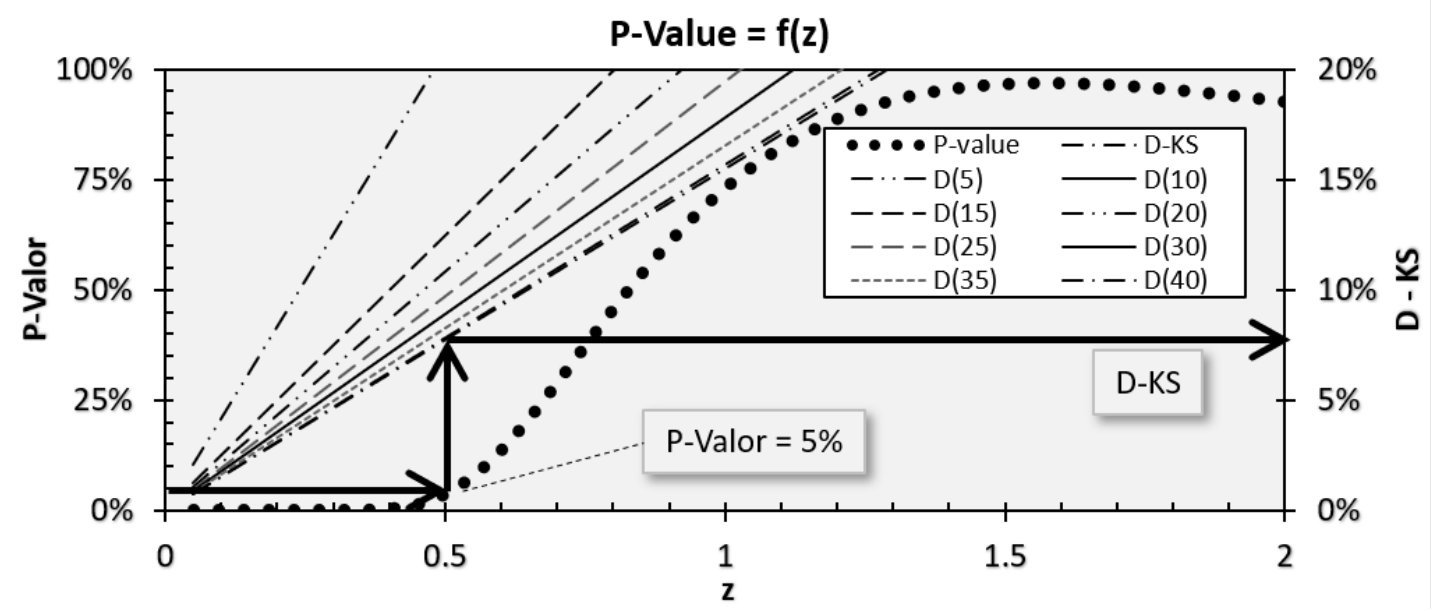

Figura 3 - P-Valor em função da variável z e da estatística K-S para tamanhos de amostras múltiplos de 5.

O teste de hipótese é considerado aceito se o valor de $\mathrm{D}$ calculado pela Eq. 13 for inferior ou igual ao valor crítico, obtido em função do nível de significância e do tamanho da amostra. (PRESS et al., 2007).

\subsubsection{Coeficiente de determinação}

Outra forma de analisar os dados é comparar o ajuste por mínimos quadrados das precipitações observadas em relação às modeladas pelas distribuições teóricas. As Eq. 16, 17, 18 e 19 representam o sistema de equações para a determinação dos coeficientes de ajuste por mínimos quadrados e sua respectiva qualidade (LEVINE et al., 2008).

$$
\mathrm{q}=\sum_{\mathrm{i}=1}^{\mathrm{n}}\left(\mathrm{P}_{\mathrm{i}}-\mathrm{a}-\mathrm{b} \times \mathrm{P}_{\mathrm{m}, \mathrm{i}}\right)^{2}
$$

$$
\frac{\partial \mathrm{q}}{\partial \mathrm{a}}=-2 \sum_{\mathrm{i}=1}^{\mathrm{n}}\left(\mathrm{P}_{\mathrm{i}}-\mathrm{a}-\mathrm{P}_{\mathrm{m}, \mathrm{i}}\right)=0
$$

$$
\frac{\partial q}{\partial b}=-2 \sum_{i=1}^{n} P_{m}\left(P_{i}-a-b \times P_{m, i}\right)=0
$$


$\mathrm{R}^{2}=\frac{\sum_{\mathrm{i}=1}^{\mathrm{n}}\left(\mathrm{a}+\mathrm{b} \times \mathrm{P}_{\mathrm{m}, \mathrm{i}}-\overline{\mathrm{P}}_{\mathrm{l}}\right)}{\sum_{\mathrm{i}=1}^{\mathrm{n}}\left(\mathrm{P}_{\mathrm{i}}-\overline{\mathrm{P}}_{1}\right)^{2}}$

Em que: q é a soma dos quadrados dos desvios; $P_{i}$ é a precipitação observada de ordem i; $P_{m, i}$ é a precipitação modelada pela distribuição teórica; a e b são os coeficientes lineares e angulares do ajuste da reta dos dados observados em função dos dados modelados, respectivamente.

\subsection{Desagregação dos valores máximos diários em subdiários}

Com a melhor combinação de probabilidade empírica $x$ teórica foram calculadas as precipitações máximas para tempos de retorno desejáveis. Entretanto, quando se trata de dados diários de precipitação, é necessário fazer a desagregação em valores subdiários. Desse modo, coeficientes propostos pela Cetesb (1979) foram utilizados, devido à ausência de dados disponíveis. A Tabela 2 representa esses coeficientes.

Tabela 2 - Coeficientes de desagregação

\begin{tabular}{|c|c|c|c|c|c|}
\hline $\mathbf{t 1} \rightarrow \mathbf{t 2}$ & Coeficiente & $\mathbf{t 1} \rightarrow \mathbf{t 2}$ & Coeficiente & Coeficiente \\
\hline $24 \mathrm{~h} / 1 \mathrm{dia}$ & 1,14 & $6 \mathrm{~h} / 24 \mathrm{~h}$ & 0,42 & $20 \mathrm{~min} / 30 \mathrm{~min}$ \\
\hline $12 \mathrm{~h} / 24 \mathrm{~h}$ & 0,85 & $1 \mathrm{~h} / 24 \mathrm{~h}$ & 0,74 & $15 \mathrm{~min} / 30 \mathrm{~min}$ \\
\hline $10 \mathrm{~h} / 24 \mathrm{~h}$ & 0,82 & $30 \mathrm{~min} / 1 \mathrm{~h}$ & 0,74 & 0,7 \\
\hline $8 \mathrm{~h} / 24 \mathrm{~h}$ & 0,78 & $25 \mathrm{~min} / 30 \mathrm{~min}$ & 0,91 & 0,54 \\
\hline
\end{tabular}

Fonte: CETESB (1979).

\subsection{Determinação dos coeficientes da IDF tipo Sherman}

A Equação IDF tipo Sherman possui o formato da Eq. 20.

$i=\frac{K \cdot R^{a}}{(b+t)^{c}}$

Em que: $\mathrm{i}=$ intensidade em $\mathrm{mm} / \mathrm{h} ; \mathrm{TR}=$ tempo de retorno em anos; $t$ = duração em minutos; $K, a$, b, c são parâmetros adimensionais obtidos pelo ajuste.

A determinação dos parâmetros $\mathrm{K}$, $\mathrm{a}$, $\mathrm{b}$ e c pode ser feita utilizando a ferramenta solver do Micro- soft Excel $®$. Por outro lado, uma outra abordagem é a linearização da Eq. 20, que fornece um sistema de equações para a determinação de $K$, a e c (BRITTO; KELLNER, 2016). Nessa abordagem, para a solução do sistema de equações é necessária a adoção do parâmetro b (CAVALCANTI, SILVA E REIS, 2015).

A obtenção do parâmetro $b$ foi realizada utilizando o solver do Excel $®$ pelo método GRG não linear, por meio da minimização da Eq. 13, ou seja, após admitir um valor de $b$, os outros três parâmetros são calculados pela solução do sistema de equações. Esse processo se repete até que se tenha a minimização da função objetivo (Eq. 21).

$\min \mathrm{F} . \mathrm{O}=\sqrt{\frac{\sum_{\mathrm{TR}=\mathrm{TR}_{1}}^{\mathrm{TR}} \sum_{\mathrm{t}=\mathrm{t}_{1}}^{\mathrm{t}_{\mathrm{m}}}\left(\mathrm{P}_{\mathrm{aj} . \mathrm{ad}, \mathrm{TR}, \mathrm{t}_{\mathrm{i}}}-\mathrm{P}_{\mathrm{mod}, \mathrm{TR}, \mathrm{t}_{\mathrm{i}}}\right)^{2}}{\mathrm{~m} \cdot \mathrm{n}}}$ 
Onde $P_{\text {aj.ad,TR,ti }}$ é a precipitação para o ajuste adotado desagregada em ti, para dado TR; $\mathrm{P}_{\text {modl,TR,ti }}$ é a precipitação modelada com os parâmetros da eq. de Sherman, m é o número de intervalos de tempo adotados na desagregação e n é o número de tempos de retorno adotado no cálculo.

\section{8 Ábacos de projeto de estruturas hidráulicas}

A aplicação do método racional a bacias pequenas é difundida mundialmente, conforme apresentado por Canholi (2005), Tucci (2012) e Akan
(1993). Uma forma eficiente e de aplicação prática para a determinação das vazões máximas de projetos nesse tipo de bacia é a combinação do método SCS-CN (1986) com o Método Racional. Uma abordagem detalhada do método é encontrada em Gomes Júnior et al., (2019). Assim, conhecendo o uso e ocupação da bacia, tempo de concentração e risco admitido no projeto, podese elaborar gráficos para determinar as vazões de projeto de determinada região onde a IDF é válida. A Eq. 22 apresenta a função que descreve a vazão específica de projeto.

$q\left(T R, C N, t_{c}\right)=C . i=\frac{5}{18}\left[\frac{\left(\frac{K . R^{a}}{\left(b+t_{c}\right)^{c}} \times \frac{t_{c}}{60}-\frac{5080}{C N}-50,8\right)^{2}}{\left(\frac{K . R^{a}}{\left(b+t_{c}\right)^{c}} \times \frac{t_{c}}{60}+\frac{20320}{C N}-203,2\right) t_{c}}\right]$

Onde q é a vazão específica $\left(\mathrm{m}^{3} / \mathrm{s} / \mathrm{km}^{2}\right)$, $\mathrm{t}_{\mathrm{c}}$ é o tempo de concentração da bacia (min), $\mathrm{CN}$ é o coeficiente curve-number da condição hidrológica de projeto, Q é a vazão de projeto $\left(\mathrm{m}^{3} / \mathrm{s}\right)$, A é a área da bacia $\left(\mathrm{km}^{2}\right)$, C é o coeficiente de runoff e i é a intensidade de precipitação em $\mathrm{mm} / \mathrm{h}$.

\section{RESULTADOS E DISCUSSÃO}

A estação pluviométrica No 83.726 possuía 57 anos de dados de precipitações diárias, de 01/01/1961 até 31/12/2018.

\subsection{Remoção de Outliers}

Foram removidos 11 anos de dados de precipitação por possuírem mais de 10 falhas ao longo de todo o ano e mais seis anos por possuírem pelo menos uma falha durante o período chuvoso.
Após a filtragem dos anos que passaram nesses critérios, foi elaborada uma análise de frequência, obtendo-se os valores máximos anuais de precipitação para cada ano. A aplicação do método de remoção de outliers descrito nas Eq. 1, 2 e 3 permitiu concluir que um dado de precipitação fora considerado outlier. Com isso, restaram 39 precipitações máximas anuais para a construção da Equação IDF.

\subsection{Histograma de Frequências}

\subsubsection{Construção do histograma de frequências e análise prévia visual}

Os dados observados e modelados foram analisados por meio de um histograma de frequências. Na Tabela 3 são apresentados alguns valores resultantes da análise estatística descritiva da amostra. 
Tabela 3 - Descrição estatística dos dados tratados de precipitação em São Carlos - SP

\begin{tabular}{|c|c|c|c|c|c|c|c|}
\hline Variável & Valor & Variável & Valor & Variável & Valor & Variável \\
\hline $\mathbf{N}$ & 39 & $\boldsymbol{\sigma}_{\mathbf{P}}$ & $15.5 \mathrm{~mm}$ & $\mathbf{C}_{\mathbf{v}}$ & 0.193 & $118.9 \mathrm{~mm}$ \\
\hline$\overline{\mathbf{P}}$ & $80.5 \mathrm{~mm}$ & Intervalo & $15 \mathrm{~mm}$ & $\mathbf{G}$ & +0.623 & $\mathbf{P}_{\text {máx }}$ \\
\hline
\end{tabular}

Onde N é o tamanho da amostra, $\overline{\mathrm{P}}$ é a precipitação diária máxima média anual, $\sigma_{\mathrm{p}}$ é o desvio padrão da amostra, Intervalo é o tamanho dos intervalos do histograma de frequência construído, $C_{v}$ é o coeficiente de variação, $G$ é o coeficiente de assimetria e $P_{\operatorname{máx}}$ e $P_{\min }$ são as precipitações máximas e mínimas anuais, respectivamente.

São observados valores máximos de precipitação diária da ordem de $115 \mathrm{~mm}$. Contudo, para a extrapolação dos dados para análises de distribuição de probabilidades, deve-se avaliar o ajuste de cada distribuição de acordo com os dados. Assim, definindo 9 intervalos de precipitação, ou seja, faixas de $15 \mathrm{~mm}$ em $15 \mathrm{~mm}$, as precipitações foram agrupadas e foi construído o histograma de frequências apresentado na Fig. 4. Além disso, utilizando as funções de distribuição de probabilidades de cada método (Normal, Gumbel e Log-Normal), foram plotados os histo- gramas teóricos correspondentes ao histograma de frequências reais, conforme ilustra a Fig. 4.

\subsubsection{Análises do tempo de retorno para as precipitações observadas}

Além da utilização de histogramas para avaliar o impacto das distribuições de frequência empíricas no ajuste de distribuições teóricas, foi realizada a construção de um gráfico que relaciona a precipitação com o tempo de retorno do evento. A Fig. 5 apresenta a relação entre as distribuições de frequência empíricas e teóricas para os mesmos valores de precipitação.

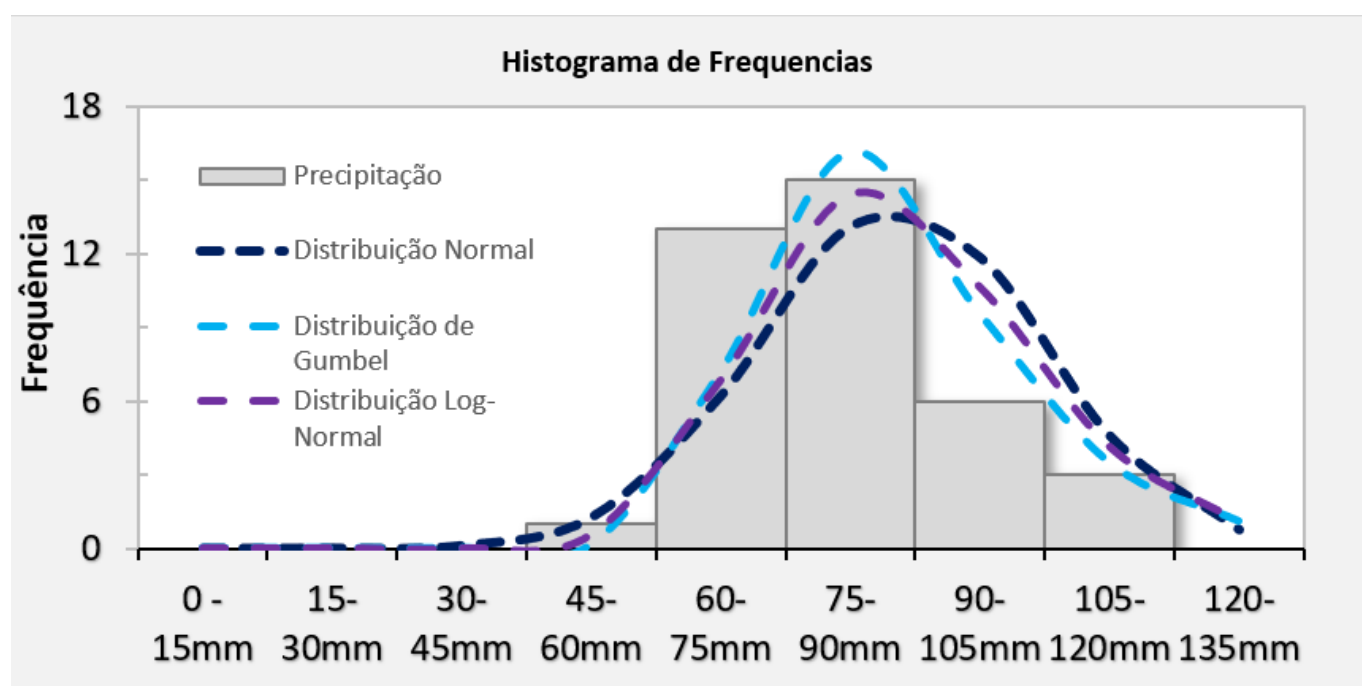

Figura 4 - Histograma de Frequências com intervalos de $15 \mathrm{em} 15 \mathrm{~mm}$ para as distribuições (a) Normal, (b) Gumbel e (c) Log-normal 


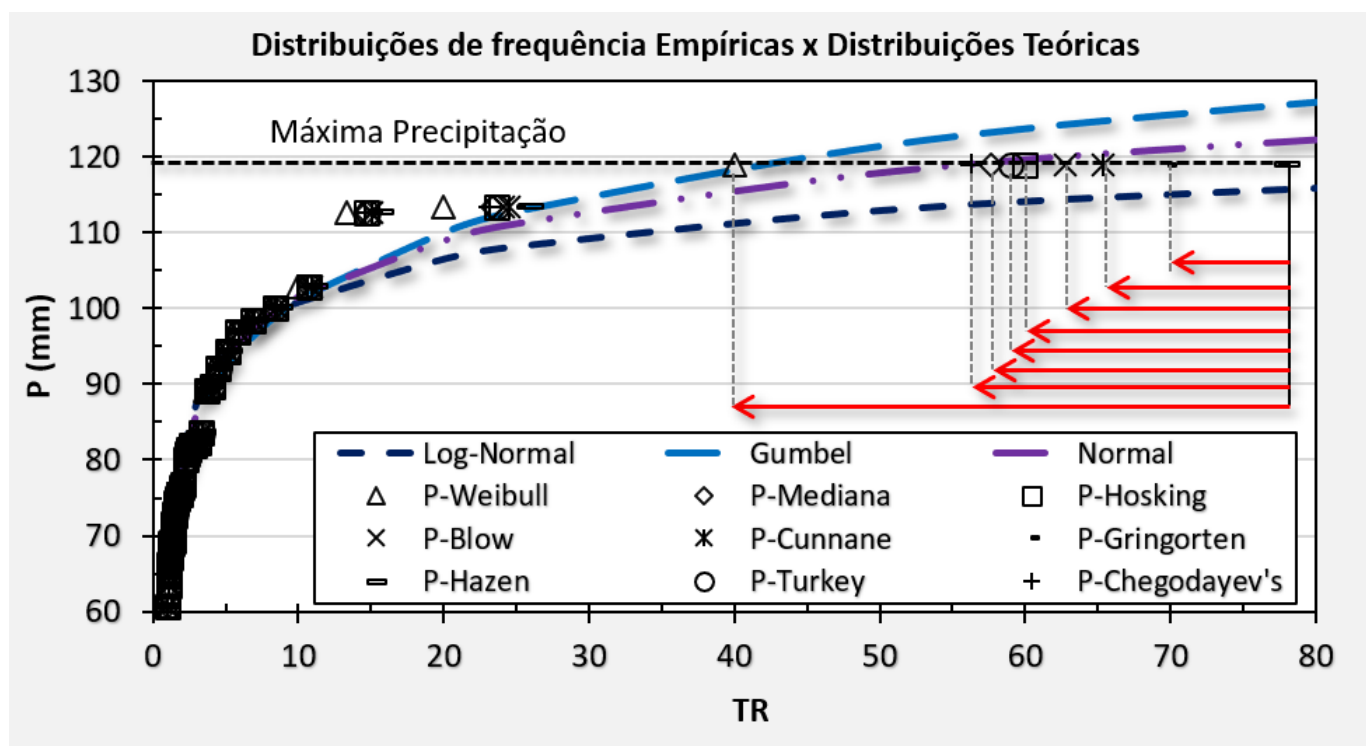

Figura 5 - Comparação entre os métodos empíricos e teóricos em relação ao tempo de retorno para os dados tratados de precipitação em São Carlos - SP.

A análise dos dados permitiu observar, para cada um dos 39 valores de precipitações utilizados, quais frequências empíricas, isto é, quais tempos de retorno, são associadas a cada um dos valores. Observam-se menores tempos de retorno para a fórmula empírica de Weibull, sendo o seu máximo valor da ordem de 40 anos de tempo de recorrência. Por outro lado, a probabilidade empírica estimada pelo método de Hazen é a que apresenta, para os mesmos valores de precipitação, tempos de retorno maiores.
Para o evento máximo observado $(P \cong 120 \mathrm{~mm})$, nota-se uma faixa de variação em tempos de recorrência varrendo desde 40 anos a algo próximo a 80 anos, o que representa um acréscimo de aproximadamente $100 \%$ no tempo de retorno dependendo do método empírico. A relação entre as frequências acumuladas empíricas e teóricas acumuladas é apresentada na Fig. 6, que serviu de base para a estimativa da estatística D, Eq. 13.

Figura 6 - Análises gráficas do teste de Kolmogorov-Smirnov de cada distribuição empírica em relação as distribuições teóricas de Gumbel, Normal e Log-Normal

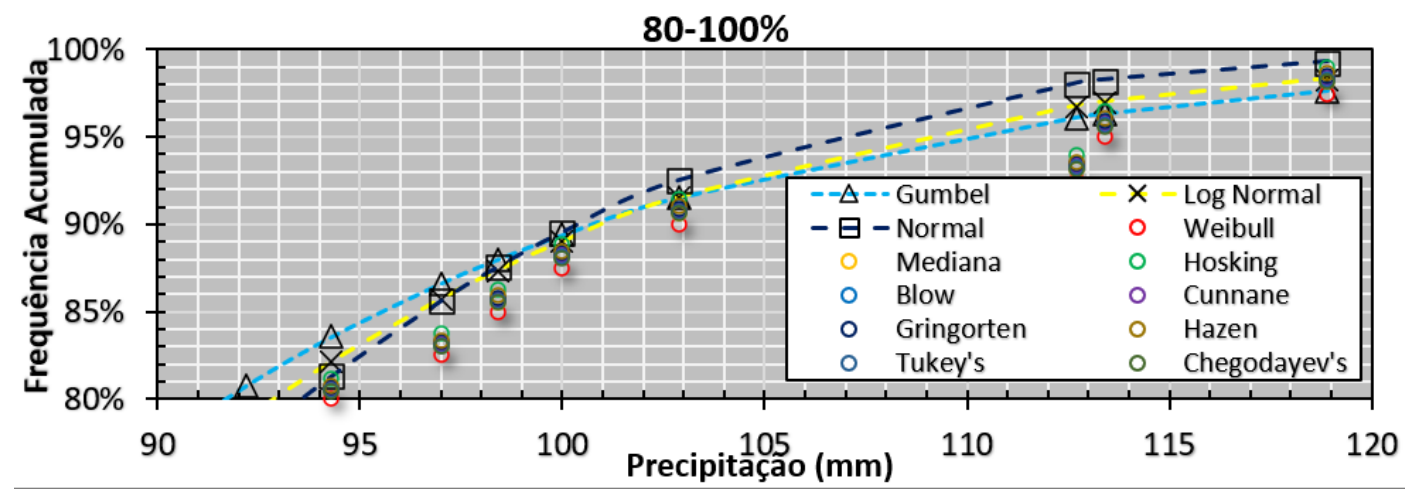

continua... 
Figura 6 - Continuação...
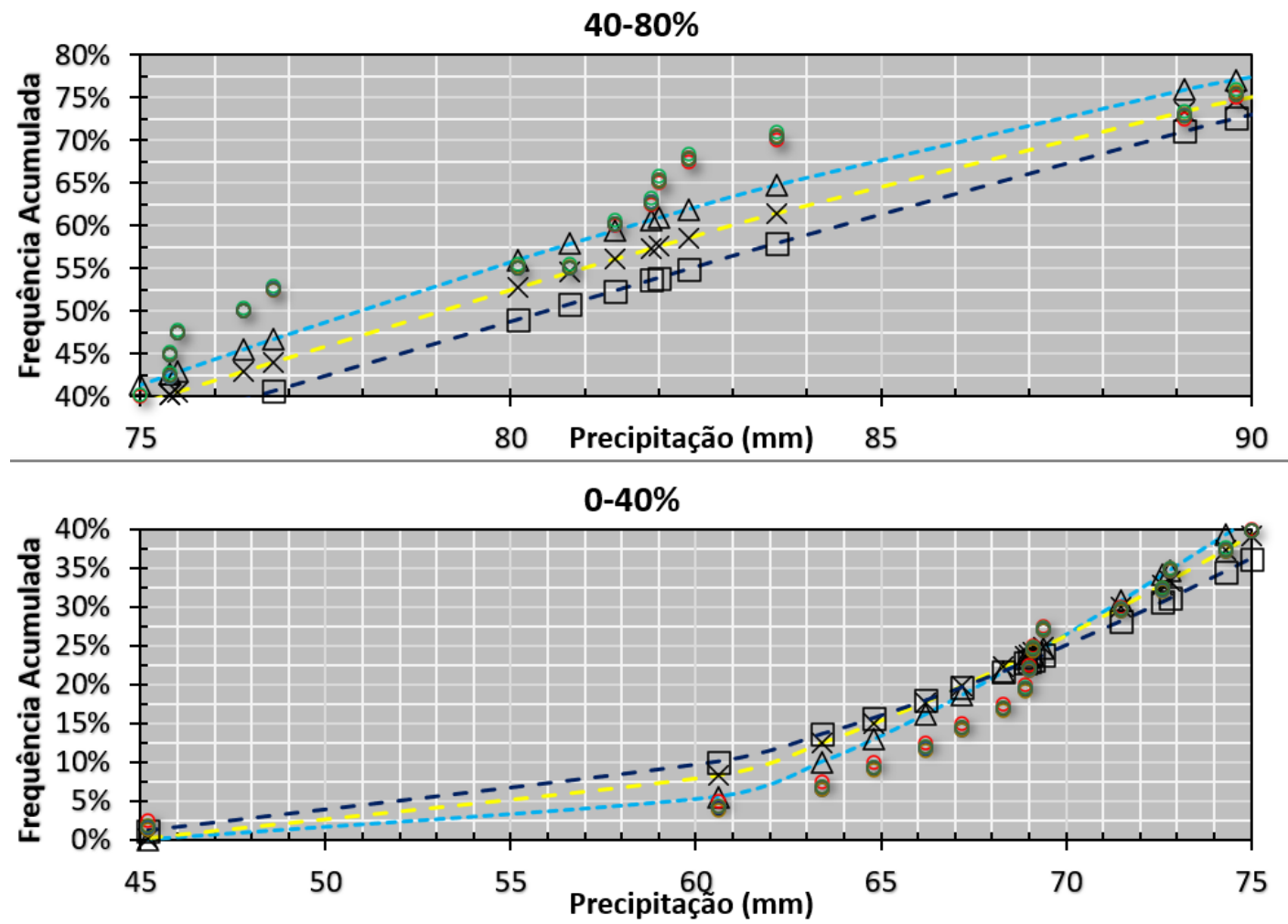

\subsection{Análises Estatísticas}

Os resultados da análise de cada método empírico com cada método teórico analisado são apresentados na Tabela 4.

Tabela 4 - Resultados obtidos das análises feitas combinando cada método empírico versus

\begin{tabular}{|c|c|c|c|c|c|c|c|c|c|}
\hline \multirow{2}{*}{ Empírico } & \multicolumn{3}{|c|}{ Gumbel } & \multicolumn{3}{|c|}{ Log Normal } & \multicolumn{3}{c|}{ Normal } \\
\hline Weibull & $\mathbf{R}$ & $\mathbf{R}$ D-KS & P-valor & $\mathbf{R}^{2}$ & D-KS & P-valor & $\mathbf{R}^{2}$ & D-KS & P-valor \\
\hline Mediana & $98.26 \%$ & $8.3 \%$ & $5.83 \%$ & $97.41 \%$ & $11.4 \%$ & $33.51 \%$ & $94.81 \%$ & $15.1 \%$ & $68.91 \%$ \\
\hline Hosking & $98.12 \%$ & $8.4 \%$ & $6.40 \%$ & $97.24 \%$ & $11.8 \%$ & $37.81 \%$ & $94.70 \%$ & $15.4 \%$ & $71.12 \%$ \\
\hline Blow & $97.63 \%$ & $8.2 \%$ & $5.30 \%$ & $97.62 \%$ & $11.6 \%$ & $35.66 \%$ & $95.20 \%$ & $15.2 \%$ & $69.66 \%$ \\
\hline Cunnane & $97.56 \%$ & $8.5 \%$ & $6.99 \%$ & $97.19 \%$ & $11.8 \%$ & $37.81 \%$ & $94.68 \%$ & $15.5 \%$ & $71.84 \%$ \\
\hline Gringorten & $97.42 \%$ & $8.5 \%$ & $6.99 \%$ & $97.16 \%$ & $11.8 \%$ & $37.81 \%$ & $94.66 \%$ & $15.5 \%$ & $71.84 \%$ \\
\hline Hazen & $97.17 \%$ & $8.6 \%$ & $7.61 \%$ & $97.12 \%$ & $11.9 \%$ & $38.89 \%$ & $94.65 \%$ & $15.5 \%$ & $71.84 \%$ \\
\hline Tukey's & $97.74 \%$ & $8.4 \%$ & $6.40 \%$ & $97.22 \%$ & $11.8 \%$ & $37.81 \%$ & $94.69 \%$ & $15.4 \%$ & $71.12 \%$ \\
\hline Chegodayev's & $97.82 \%$ & $8.4 \%$ & $6.40 \%$ & $97.25 \%$ & $11.7 \%$ & $36.74 \%$ & $94.71 \%$ & $15.4 \%$ & $71.12 \%$ \\
\hline
\end{tabular}

Todas as combinações de distribuições passaram no teste de Kolmogorov-Smirnov para um nível de significância de $5 \%$, pois o $D_{\text {crítico }}$ para uma amostra de 39 elementos é de $21 \%$. Entretanto, ao avaliar a estatística $D$ de Kolmogorov-Smir- nov dada pela Eq. 13, apenas a distribuição de Gumbel é aceita para um nível de significância de $10 \%$. Desse modo, observa-se que as quatro melhores combinações foram Gumbel-Hosking, Gumbel-Weibull e Gumbel-Tukey's. 
4.4 Ajuste das curvas IDF para durações de 5 a 60 min e 60 a 1440 min

Como a combinação da frequência acumulada empírica de Weibull com a distribuição teórica de Gumbel foi a de melhor coeficiente de determinação e foi aprovada com no teste K-S, ela foi adotada para a elaboração dos ajustes matemáticos da Equação IDF tipo Sherman. A construção do ajuste se deu para as durações de $5,10,15$, $20,25,30,60,360,480,720$ e 1440 min para os tempos de retorno de 2, 5, 10, 25, 50, 100 e 500 anos. Os parâmetros obtidos possibilitaram expressar a Equação IDF como:

$$
i=\frac{819,67 \cdot \mathrm{TR}^{0,138}}{(10,77+\mathrm{t})^{0,75}}
$$

Supondo uma pressão atmosférica de 0,9ATM, uma coluna de fluido de $12 \mathrm{~km}$ e uma temperatura inicial de $40^{\circ} \mathrm{C}$, a coluna de água precipitável $\left(h_{p}\right)$ é de cerca de $140 \mathrm{~mm}$ supondo decaimento de temperatura linear de $-6,5^{\circ} \mathrm{C} / \mathrm{km}$ ( $\mathrm{CHOW}$ et al., 1998) e considerando a constante dos gases ideais de 287J/(kg.K). Na Fig. 7 são apresentadas as curvas IDF e PDF de melhor ajuste.

Figura 7 - Curvas IDF e PDF atualizadas para a cidade de São Carlos de 5 a 60 min e de 60 a 1440 min

\section{Curva IDF (5-60min)}
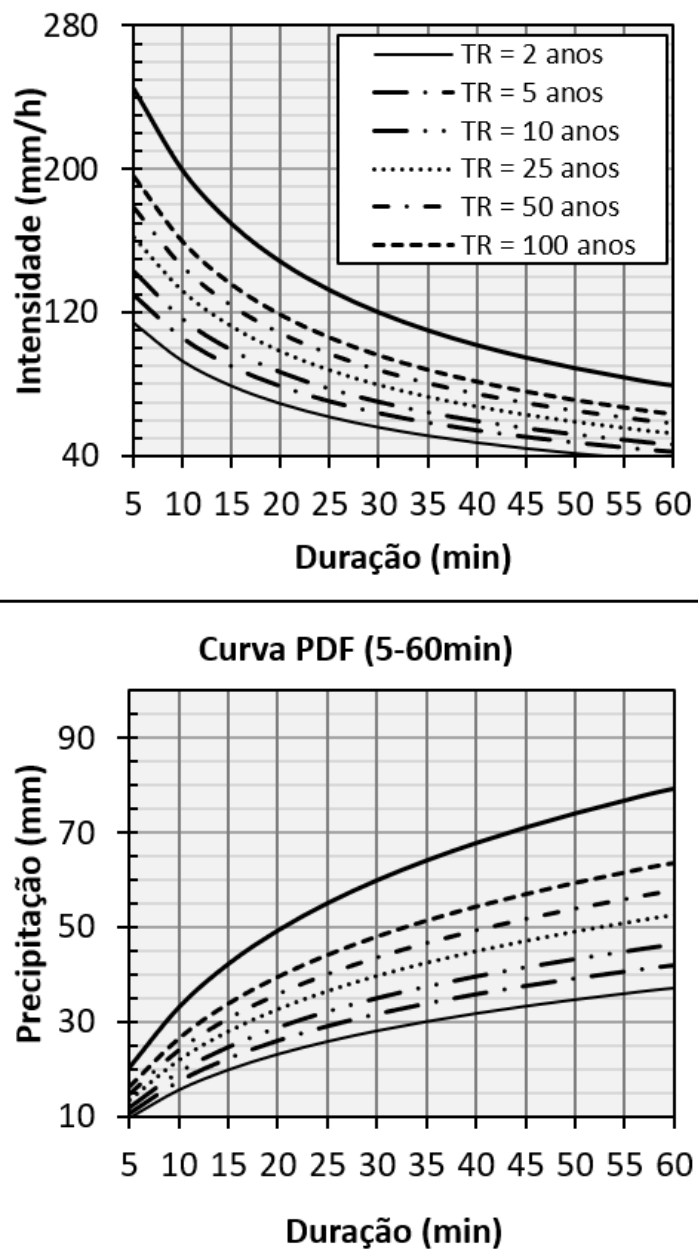

Curva IDF (60-1440min)

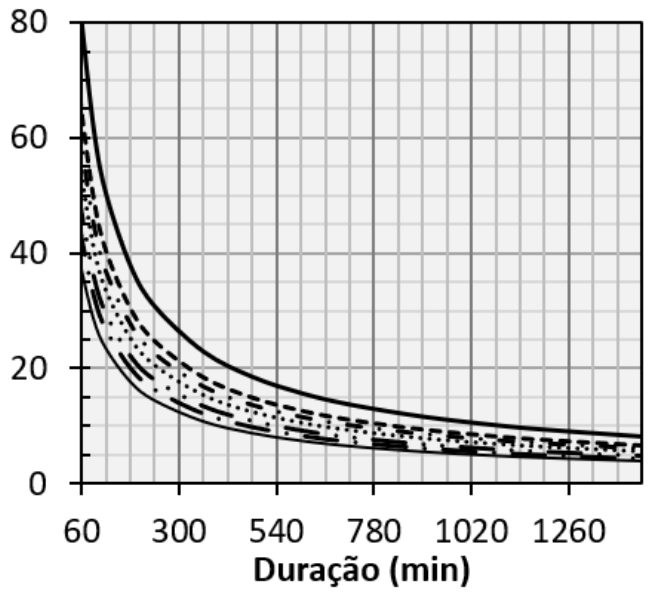

Curva PDF (60-1440min)

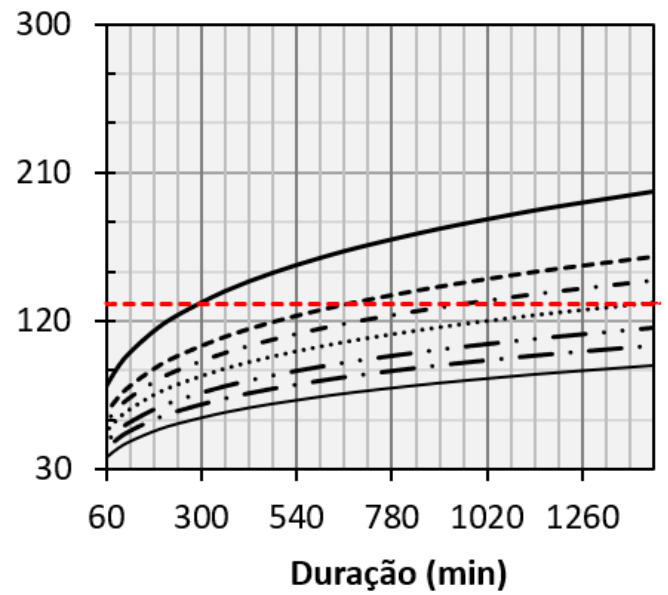

Onde a linha vermelha tracejada representa a máxima coluna de água precipitável. 
Aplicando a Eq. 22 para curve-numbers de 75, $80,85,90,95$ e 98 (i.e., curve numbers usuais para bacias urbanas) e para os tempos de retorno de 2, 5, 10, 25, 50 e 100, obtêm-se os gráficos de descarga específica de projeto para a cidade de São Carlos, conforme ilustra a Fig. 8.

Figura 8 - Ábacos de dimensionamento de pequenas obras hidráulicas para a cidade de São Carlos - SP.
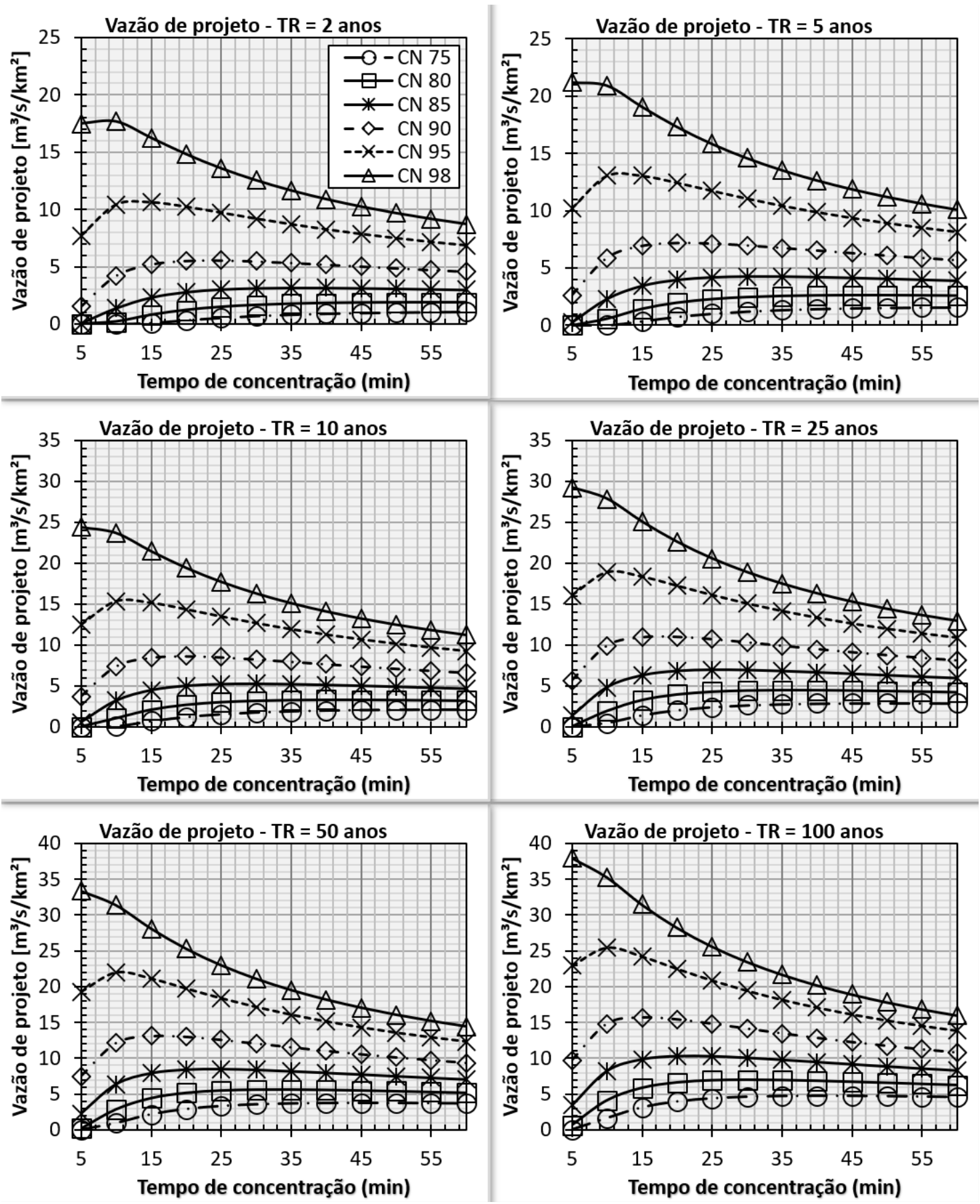


\section{DISCUSSÕES}

Embora todas as combinações de distribuições teóricas-empíricas tenham passado no teste $\mathrm{K}-\mathrm{S}$ com um nível de significância de $5 \%$, ao analisar a probabilidade do valor da estatística D ter sido obtida ao viés, nenhuma das combinações atendeu a um nível de significância de $5 \%$. Por outro lado, a distribuição de Gumbel apresentou o melhor coeficiente de determinação com os dados observados. As distribuições empíricas de Weibull e a de Hazen apresentaram uma variação de mais de $100 \%$ no tempo de retorno estimado para o maior evento (Fig. 5). Isso pode gerar diferenças significativas em obras hidráulicas projetadas com os diferentes ajustes. Um exemplo similar dessa variação é apresentado em Collischonn e Dornelles (2013).

Embora a distribuição de Weibull aparentemente superestime alguns eventos e tenha um bom ajuste com a distribuição de Gumbel, ela é fundamentada pela teoria dos grandes números (BERNOULLI, 1713) e tende a se ajustar meIhor para grandes amostras. Desse modo, optou-se por sua utilização em detrimento da de Hosking, que embora apresentasse menor valor da estatística $D$ e de $P$-Valor, tinha menor valor de coeficiente de determinação.

O critério de outliers utilizado é, de certa forma, relativamente restritivo. Mudanças razoáveis nas curvas IDFs podem ser observadas considerando os valores extremos sem o tratamento de outliers. Um ponto importante é a relevância física do problema, que está fundamentada por condições climatológicas, isto é, algumas precipitações extremas extrapoladas por métodos probabilísticos teóricos tendem a superar condições de risco ínfimo como a condição da máxima coluna d'água precipitável. Por isso, torna-se importante avaliar essa grandeza e compará-la com as precipitações extrapoladas por ajustes de probabilidades teóricos. Eviden- temente, há incertezas na avaliação e determinação da coluna de água precipitável; entretanto, ela é uma informação relevante e pode ser utilizada como uma restrição superior em curvas IDFs, especialmente na ausência de grandes séries históricas de precipitação.

É possível inferir da Fig. 7 uma pequena variação entre os métodos Mediana, Blow, Tukey's e Chegodayev's, sendo a escolha de um ou outro sem grandes variações potenciais para a amostra de precipitações observadas. Isso pode ser explicado pela proximidade dos valores b da Eq. 5 .

Muito embora as distribuições Normal e Log-Normal não sejam as mais adequadas para valores extremos, todas passaram no teste $\mathrm{K}-\mathrm{S}$ e é interessante avaliar que ambas tiveram os melhores ajustes às distribuições empíricas de Weibull e Hosking, o que sugere que as duas distribuições são as mais aplicáveis aos dados analisados independentemente das distribuições teóricas escolhidas.

A cidade de São Carlos - SP, historicamente, sofre com alagamentos e inundações. Desse modo, uma das formas de abordar esse problema é utilizar técnicas compensatórias em drenagem urbana. 0 dimensionamento dessas, por sua vez, necessita de dados de precipitação, portanto a utilização de curvas IDF é uma ferramenta importante para o manejo dos riscos de enchentes, justamente por servir de base para os seus projetos. Por outro lado, a decisão de qual método empírico usar para se basear no ajuste matemático da curva pode também ser feita levando em conta critérios de planejamento e manejo dos recursos hídricos. Assim, a escolha dos métodos que superestimam as precipitações pode representar uma margem de segurança para projetos de drenagem urbana.

A distribuição de Hazen foi a que apresentou o pior desempenho, levando em conta tanto o co- 
eficiente de determinação como a estatística de K-S e seu P-Valor apresentou, em linhas gerais, para o mesmo valor de precipitação, os maiores tempos de retorno. É interessante pensar que seu uso é utilizado com frequência na França, onde o conceito de técnicas compensatórias em drenagem urbana é bem desenvolvido e os projetos de drenagem não precisam atender a grandes eventos para determinado risco admitido, haja vista a descentralização das técnicas e o desenho usualmente relativamente mais resiliente de suas cidades (BAPTISTA, NASCIMENTO E BARRAUD, 2011).

Em relação às incertezas, é importante notar que o próprio ajuste da Equação IDF no formato tipo Sherman introduz algum tipo de erro. Além disso, a utilização dos coeficientes de desagregação propostos pela Cetesb (1979) tem grande sensibilidade no cálculo dos valores de precipitação subdiários.

Os ábacos de dimensionamento são ferramentas práticas que fornecem vazões de pico em função de critérios hidrológicos e de uso e ocupação básicos, como o curve-number, que pode ser encontrado em livros e tabelas (e.g., Canholi (2005), SCS (1986)), tempo de concentração (e.g., Silveira (2005)) e risco admitido, que tipicamente é uma grandeza estabelecida pelos órgãos reguladores do munícipio e/ou estado.

\section{CONCLUSÕES}

As distribuições de frequência empíricas, quando comparadas visando ao ajuste com distribuições teóricas, apresentam diferenças significativas na análise do tempo de retorno de uma determinada intensidade máxima média de precipitação. Os resultados obtidos permitem concluir que, para os dados da cidade de São Carlos, a distribuição de valores extremos Gumbel mais se adequa às distribuições de fre- quência empíricas de Weibull e Hosking. A utilização de curvas IDF atualizadas em São Carlos pode auxiliar no desenho de novas estruturas de drenagem para o controle dos escoamentos. A utilização dos ábacos simplifica e otimiza o cálculo das vazões de projeto e pode ser apresentada em planos diretores como guia de dimensionamento de estruturas de drenagem urbana.

Recomenda-se em trabalhos futuros avaliar estatisticamente as combinações entre métodos teóricos-empíricos de probabilidades para regimes de precipitações menos e mais extremos em relação a São Carlos - SP. Além disso, a utilização de mais distribuições de probabilidade teóricas pode ser incluída na análise, gerando mais combinações de distribuições teóricas e empíricas e, portanto, mais flexibilidade para o ajuste dos dados. Por fim, a metodologia aplicada neste texto é abrangente e pode ser aplicada para a elaboração de ábacos de vazão específica e IDFs, podendo ser replicada em estudos hidrológicos.

\section{AGRADECIMENTOS}

Os autores agradecem à Fundação de Amparo à Pesquisa do Estado de São Paulo (Fapesp) processo - 2018/20865-0 e ao CNPq pelo incentivo financeiro às pesquisas.

\section{CONTRIBUIÇÃO DOS AUTORES}

Organização, Redação, Obtenção dos resultados, Compatibilização das alterações e sugestões: Gomes Júnior MN; Revisão bibliográfica, Investigação dos métodos disponíveis, Revisão da primeira versão, levantamento de dados: Braga PH; Revisão e sugestões de melhoria da primeira versão: Reis LFR, Mendiondo EM. 


\section{REFERÊNCIAS}

ADIB, A.; GHAFARI RAD, S. Development of a new integrated method for generation IDF curves based on three climatic changes scenarios. Scientia Iranica, v. 26, n. 2, p. 742-751, 2017. https://doi.org/10.24200/sci.2017.4593

AKAN, O. A. Urban stormwater hydrology: a guide to engineering calculations. Crc Press, 1993.

BACK, Á. J.; OLIVEIRA, J. L. R.; HENN, A. Relações entre precipitações intensas de diferentes durações para desagregação da chuva diária em Santa Catarina. Revista Brasileira de Engenharia Agrícola e Ambiental, v. 16, n. 4, p. 391-398, abr. 2012. https://doi.org/10.1590/S1415-43662012000400009

BAPTISTA, M. B.; NASCIMENTO, N. O.; BARRAUD, S. Técnicas compensatórias em drenagem urbana. ABRH, 2011.

BATISTA, T. L.; ALVES, F. DE M.; TAVARES, P. R. L. Utilização de dados pluviométricos para o desenvolvimento de equações idf da região metropolitana de Fortaleza-CE, Brasil. Revista DAE, v. 66, n. 211, p. 118-129, 2018. https://doi.org/10.4322/ dae.2018.018

BLOW, G. Statistical Estimates and Transformed Beta-Variables. 1. ed. New York: John Wiley \& Sons, 1958.

BRITTO, L. N.; KELLNER, E. Procedimento para Construção da Equação de Chuva a partir das máximas precipitações diárias obtidas de séries anuais: Atualização da Equação de Chuva da cidade de São Carlos (SP). Revista Nacional de Gerenciamento de Cidades, v. 4, n. 26, 25 dez. 2016.

CANHOLI, A. Drenagem urbana e controle de enchentes. Oficina de textos, 2015

CAVALCANTI, D. L. O.; SILVA, D. F.; REIS, L. F. R. Análises preliminares de dados de monitoramento das bacias urbanas do município de São Carlos-SP. XXI Simpósio Brasileiro de Recursos Hídricos, p. 8, 2015. Anais...

CETESB. (1979). Drenagem urbana: manual de projeto. Departamento de Águas e Energia Elétrica (DAEE) e Companhia de Tecnologia de Saneamento (CETESB), São Paulo, 1ed., 468 p.

COLLISCHONN, W.; DORNELLES, F. Hidrologia para engenharia e ciências ambientais. Porto Alegre: Editora ABRH, 2013.

CHOW, V. T.; MAIDMENT, D. R.; MAYS, L. W. Applied Hydrology. 1. ed. [s.l.] McGraw-Hill Book Company, 1988.

CUNNANE, C. Unbiased Plotting Positions - A Review. Journal of Hydrology, v. 37, p. 205-222, 1978. https://doi. org/10.1016/0022-1694(78)90017-3

FILLIBEN, J. J. The Probability Plot Correlation Coefficient Test for Normality. Technometrics, v. 17, n. 1, p. 111-117, 1975.

GRINGORTEN, I. I. A plotting rule for extreme probability paper. Journal of Geophysical Research, v. 68, n. 3, p. 813-814, 1963. https://doi.org/10.1029/JZ068i003p00813
GUO, S. L. A discussion on unbiased plotting positions for the general extreme value distribution. Journal of Hydrology, v. 121, n. 1-4, p. 33-44, 1990. https://doi.org/10.1016/ 0022-1694(90)90223-K

HAZEN, A. Flood flows: a study of frequencies and magnitudes. 1. ed. New York: John Wiley \& Sons, 1930.

HOSKING, J. R. M.; WALLIS, J. R.; WOOD, E. F. Estimation of the Generalized Extreme-Value Distribution by the Method of Probability-Weighted Moments. Technometrics, v. 27, n. 3, p. 251-261, 1985. https://doi.org/10.1080/00401706.1985.104 88049

IBGE. Instituto de Geografia e Estatística. Panorama - Território e Ambiente. Disponível em: < https://cidades.ibge.gov. $\mathrm{br} / \mathrm{brasil} / \mathrm{sp} / \mathrm{sao}$-carlos/panorama>. Acesso em: 22 de agosto de 2019.

INMET - Instituto Nacional de Meteorologia. Disponível em <www.inmet.gov.br>. Acesso em: 22/07/19.

JONES, C. D. et al. The HadGEM2-ES implementation of CMIP5 centennial simulations. Geoscientific Model Development, v. 4, n. 3, p. 543-570, 1 jul. 2011. https://doi.org/10.5194/gmd4-543-2011

JÚNIOR, M. N. G. et al. Preliminary Design of Detention Ponds Using Specific Design Discharge and Orifice Stage Discharge Relationship For Different Climate Patterns. XXIII Simpósio Brasileiro De Recursos Hídricos, n. 23, p. 7, 2019. Anais...

LAGO, C. A. F. Impactos das mudanças climáticas sobre a drenagem urbana subtropical com técnicas compensatórias. Dissertação (Mestrado em Ciências: Engenharia Hidráulica e Saneamento). São Carlos, Universidade de São Paulo, 2018.

LEON HARTER, H. Another look at plotting positions. Communications in Statistics-Theory and Methods, v. 13, n. 13, p. 1613-1633, 1984.

LEVINE, D. M. et al. Estatística - Teoria e Aplicações. 5. ed. Rio de Janeiro: LTC, 2008.

MIGUEZ, M.; REZENDE, O.; VERÓL, A. Drenagem urbana: do projeto tradicional à sustentabilidade. 1. ed. Rio de Janeiro: Elsevier Brasil, 2016.

SANE, Y. et al. Intensity-duration-frequency (IDF) rainfall curves in Senegal. Natural Hazards and Earth System Sciences, v. 18 , n. 7 , p. 1849-1866, 2018. https://doi.org/10.5194/ nhess-18-1849-2018, 2018

SILVEIRA, A. L. L. Desempenho de fórmulas de tempo de concentração em bacias urbanas e rurais. Revista Brasileira de Recursos Hídricos, v. 10, p. 5-23, 2005.

SILVEIRA, A. L. L. Equação para os coeficientes de desagregação de chuva. Revista Brasileira de Recursos Hídricos, v. 5, n. 4, p. 143-147, 2000. 
TORRICO, J. J. T. Práticas Hidrológicas. 1. ed. Rio de Janeiro: Transcon, 1974.

TUCCI, C. E. M. et al. Hidrologia: ciência e aplicação. São Paulo: Editora UFRGS/ABRH, 2012.

TUKEY, J. W. The future of data analysis. 1. ed. Estados Unidos: Princeton University and Bell Telephone Laboratories, 1962.
USDA, SCS. Urban hydrology for small watersheds. Technical release, v. 55, p. 2-6, 1986.

WATANABE, M. et al. Improved Climate Simulation by MIROC5: Mean States, Variability, and Climate Sensitivity. Journal of Climate, v. 23, n. 23, p. 6312-6335, dez. 2010. https://doi.org/10.1175/2010JCLI3679.1 